\title{
Coronary angioplasty in pregnancy
}

\author{
N C COWAN, M A DE BELDER, M T ROTHMAN \\ From the Cardiac Department, The London Hospital, Whitechapel, London
}

SUMMARY Myocardial infarction is rare in pregnancy. A 30 year old white primigravida had an anterior infarct at 20 weeks' gestation, which was followed by troublesome angina. Coronary angiography showed a tight stenosis of the left anterior descending coronary artery. This was treated successfully by percutaneous transluminal coronary angioplasty.

\section{Case report}

A 30 year old white woman presented at 20 weeks' gestation in her first pregnancy with paraesthesiae and severe pain in her left arm and hand. This was followed by a dull feeling of intense retrosternal pressure radiating down both arms, which was associated with profuse sweating, nausea, and vomiting. There was no previous medical or drug history.

The patient had smoked 30 cigarettes a day for fourteen years. Her father and paternal uncle had died after myocardial infarction aged 39 and 40 years respectively. There were no other associated risk factors for myocardial infarction.

The patient was admitted from home directly to the coronary care unit of her local hospital. The first electrocardiogram showed unequivocal ST elevation in leads I and V2-V6. Because the symptoms persisted she was given intravenous diamorphine. While she was being examined cardiac ventricular fibrillation occurred. Cardiopulmonary resuscitation was performed and intravenous lignocaine was administered; four direct current shocks were required to restore sinus rhythm.

Four days later further typical ischaemic chest pain occurred which did not settle despite intravenous infusion of isosorbide dinitrate. She was also treated with oral nifedipine $20 \mathrm{mg}$ twice a day, atenolol $100 \mathrm{mg}$ twice a day, slow release isosorbide dinitrate $20 \mathrm{mg}$ twice a day, diazepam $2 \mathrm{mg}$ three times a day, and potassium supplements. Urgent transfer to a regional cardiac centre was arranged.

On arrival at the centre a 12 lead electrocardiogram showed diminished $R$ waves in leads V1-V4, normal ST segments, and $\mathrm{T}$ wave inversion in lead V1 only. Ultrasonography showed a normal fetus. The gestational age was estimated to be 20.5 weeks by

Requests for reprints to Dr N C Cowan, Department of Radiology, King's College Hospital, London SE5 9RS. measurement of the biparietal diameter. Coronary angiography, performed from a right brachial approach by the Sones' technique, showed a single pronounced proximal stenosis of the left anterior descending coronary artery (fig la) and some irregularity of the posterior descending branch of the right coronary artery. The left main stem and circumflex arteries were normal. The left ventriculogram showed an akinetic anterior wall (fig 2a). During the procedure the fetus was protected by positioning lead aprons behind and over the anterior wall and flanks of the patient's abdomen. The radiographic screening time was kept as short as possible. Thermoluminescent dosimetry measurements of the radiation dose received by the patient's skin showed a maximum fetal dose of $<0.55 \mathrm{mSv}$. We assumed that a completed myocardial infarct had occurred. The patient's symptoms settled and a ten day postinfarct submaximal exercise test (equivalent to completing stage 1 of the Bruce protocol) was well tolerated. There were no cardiac symptoms or ST changes and there was a satisfactory rise in blood pressure. The test was performed on the same oral medication as that given before transfer to the regional centre, and she remained on this combination on discharge.

Nine days after discharge she had further central chest pain. The patient was immediately admitted to the regional cardiac centre. The electrocardiogram showed deep T wave inversion in leads $I$, aVL, and V2-V6. Treatment with intravenous isosorbide dinitrate was started and the patient was anticoagulated with a constant infusion of intravenous heparin. The patient's chest pain continued despite full medical treatment.

Percutaneous transluminal angioplasty of the tight stenosis of the left anterior descending artery was performed via a right femoral approach with a left coronary guide catheter (USCI), a 0.018 inch high torque floppy guide wire (ACS), and a $3 \mathrm{~mm}$ dilata- 


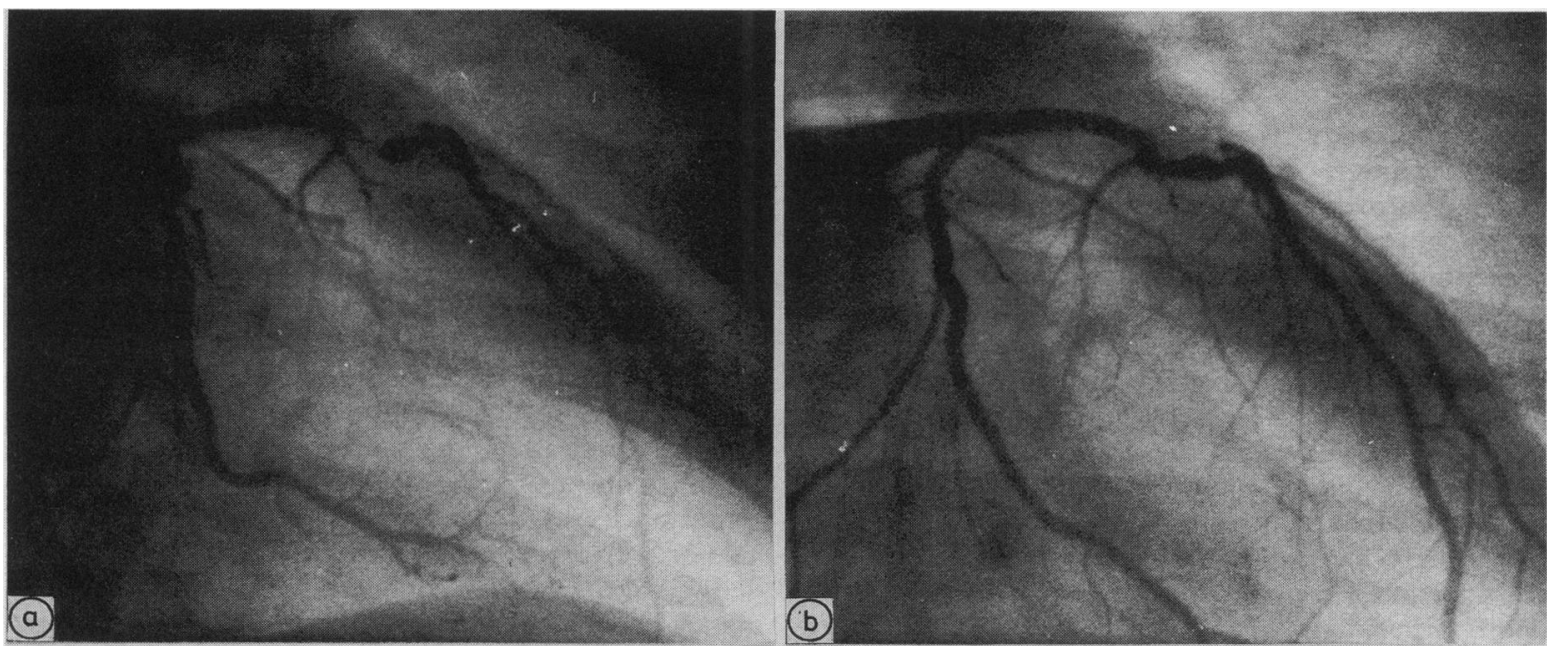

Fig 1 Right anterior oblique projections of the left anterior descending coronary artery before (a) and after (b) percutaneous transluminal coronary angioplasty.

tion catheter (ACS). Four inflations at $808 \mathrm{kPa}$ pressure were made each for 60 seconds with good result (fig $1 \mathrm{~b}$ ). $T$ wave changes were seen during the procedure but no pain was experienced. Screening time was again kept to a minimum and the fetus was shielded as for the earlier angiogram. Thermoluminescent dosimetry measurements indicated that the maximum radiation dose to the fetus was $<0.7 \mathrm{mSv}$. A constant intravenous heparin infusion was continued for $\mathbf{2 4}$ hours. After angioplasty the patient was treated with dipyridamole $100 \mathrm{mg}$ three times a day, aspirin $75 \mathrm{mg}$ once a day, diltiazem $60 \mathrm{mg}$ three times a day, and one Transiderm-Nitro patch daily (releasing $5 \mathrm{mg}$ of glyceryl trinitrate).

Two days after angioplasty the patient had an exercise test, performed according to a modified Bruce protocol; she exercised to an equivalent of one minute into stage 4 of the Bruce protocol and did not experience angina. There were no ST changes and blood pressure was well maintained.

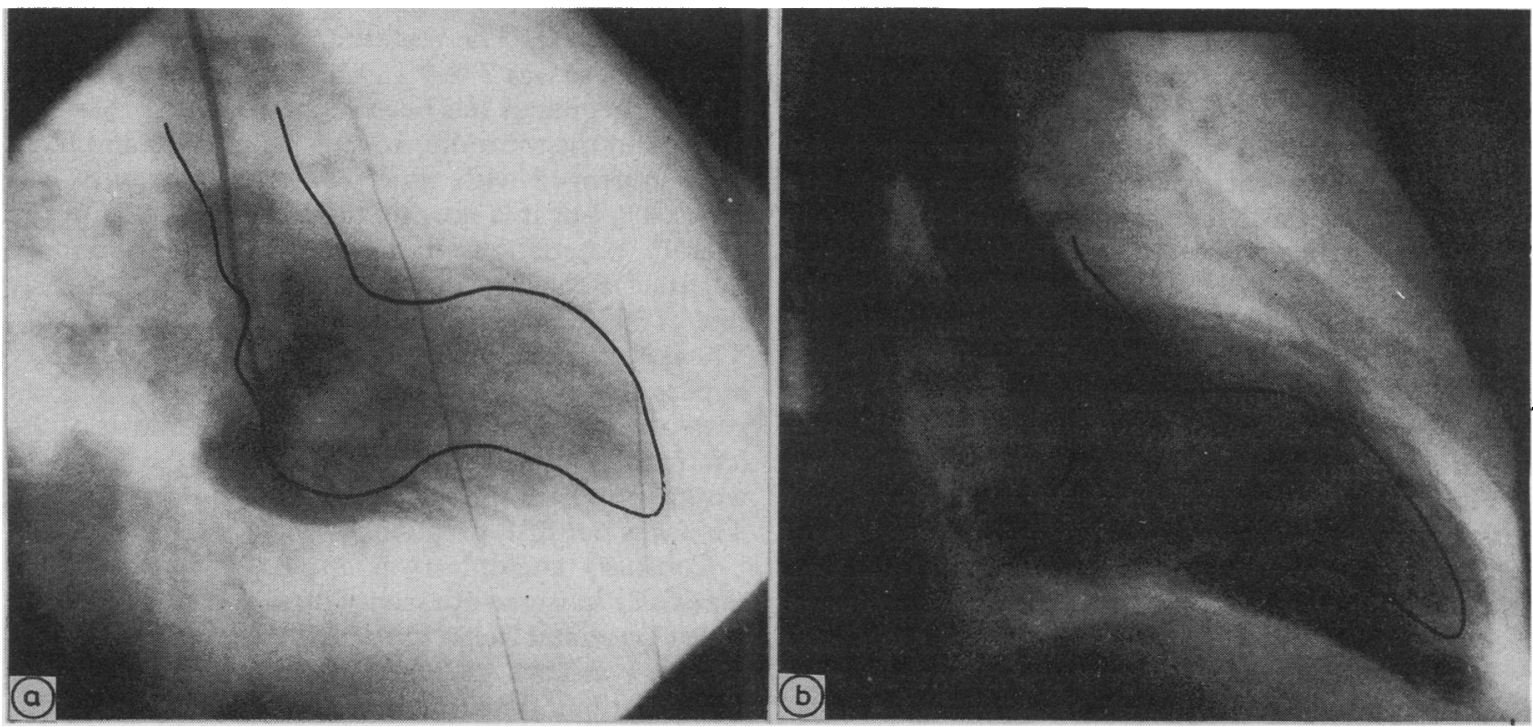

Fig 2 (a) Right anterior oblique projection of the left ventriculogram before (a) and after (b) angioplasty. Image was obtained in diastole with systolic outline superimposed. (a) Shows anterior akinesia and (b) shows improved anterior wall motion. (b) Right anterior oblique projection of the left ventriculogram in diastole with systolic outline superimposed. 
The patient was discharged home and was reviewed monthly throughout the remainder of her pregnancy, during which she was free from angina. She acted on advice to give up smoking. The electrocardiographic changes gradually reverted and the electrocardiogram was normal at delivery. At 36 weeks' gestation the fetus was found to be breech presentation. At 38 weeks an elective lower segment caesarean section was carried out under general anaesthesia. A healthy boy weighing $3.46 \mathrm{~kg}$ was delivered. Three months after the angioplasty, follow up angiography was performed at the patient's request. This showed improved anterior wall motion (fig 2b). The site of dilatation was satisfactory. After the procedure the patient used a Transiderm-Nitro patch daily for six months, remained on diltiazem and aspirin for eighteen months, and is currently still taking dipyridamole. Two years after the angioplasty both mother and child remain well. There is no evidence of diabetes mellitus or hyperlipoproteinaemia.

\section{Discussion}

We believe this to be the first report of a case of percutaneous transluminal coronary angioplasty in pregnancy. The indication for treatment of the tight stenosis of the left anterior descending coronary artery was the occurrence of angina resistant to medical treatment two weeks after an anterior myocardial infarction.

Myocardial infarction in pregnancy is rare, with a frequency which is thought to be of the order of 1 in 10000 deliveries. ${ }^{1}$ The overall mortality is probably similar to that of myocardial infarction in non-pregnant patients. It has been suggested that mortality is related to gestational age at infarction, being higher in late pregnancy when vascular stresses are at their greatest. ${ }^{2}$ Successful resuscitation from ventricular fibrillation has been reported, with maternal and fetal survival. ${ }^{23}$

The risk factors for myocardial infarction in young women include smoking, hypertension, diabetes, familial hyperlipoproteinaemia, and use of oral contraceptives. ${ }^{4}$ Of these, cigarette smoking is the most important identified risk factor. ${ }^{6}$

The choice of drug treatment for pregnant patients with symptomatic coronary artery disease must take into account any possible adverse effects of the drug on the fetus. There is no absolute contraindication to the use of a $\beta$ blocker in pregnancy; this form of treatment has been used safely in hypertensive pregnant patients. ${ }^{7}$ Early reports suggesting association with growth retardation, acute fetal distress in labour, hypoglycaemia in the newborn, and a high perinatal mortality ${ }^{8-10}$ were not confirmed by sub- sequent controlled studies. ${ }^{11}$ Although $\beta$ blockers are excreted in small amounts in breast milk, there is no evidence that this transfer is of any importance, at least in patients taking atenolol. ${ }^{12}$ There is no evidence that nitrates cause fetal abnormalities. ${ }^{13}$ All calcium antagonists cross the placenta and can inhibit uterine contractions; nifedipine and verapamil have been used as tocolytic agents in cases of premature labour and there is no evidence that these two drugs cause fetal malformations. ${ }^{14}{ }^{15}$ Diltiazem, however, remains a suspected human teratogen. ${ }^{16}$

In survivors of uncomplicated myocardial infarction in pregnancy, the method of delivery should depend on obstetric factors only. Survey of the reported cases suggests that management of the actual delivery is based more on belief than knowledge. ${ }^{1}$ Some cases of myocardial infarction in pregnancy are associated with normal angiographic appearances of the coronary arteries. ${ }^{17}$ In view of the possibility of coronary artery spasm, it seems wise not to use ergometrine immediately after delivery in survivors of myocardial infarction in pregnancy. Similarly, oxytocin infusion should be avoided if possible. ${ }^{2}$

Our patient continued to have angina after myocardial infarction. It rapidly became unstable despite further treatment and it was thought that she required intervention for the known stenosis of the left anterior descending coronary artery. The earliest reports of cardiac operation (closed mitral commissurotomy) during pregnancy appeared in 1952 ${ }^{18}$; since then several hundred cases of commissurotomy have been reported without the need for cardiopulmonary bypass. The maternal death rate was $<2 \%$ and fetal loss was $7 \cdot 5-9 \%$. Cardiopulmonary bypass during pregnancy has been reported in less than 100 cases, and the mortality results for mother and fetus have improved with time. Maternal mortality was about $4 \%$ but it is now no different from that of the overall population undergoing cardiopulmonary bypass. ${ }^{18}$ Fetal loss has been reduced from an early rate of $30 \%$, but it is still considerable (about $10 \%$ ). There is only one report, however, of coronary artery bypass surgery during pregnancy ${ }^{19}$-in a 36 year old patient with severe angina and a $90 \%$ stenosis of the left main coronary artery. The patient was 12-14 weeks' pregnant and later delivered a normal child. This was her fifth pregnancy.

Coronary angioplasty is an attractive alternative especially in a case of essentially single vessel disease with a proximal lesion in the left anterior descending coronary artery. Although there is a small risk of complications that can lead to the need for urgent operation, angioplasty does not require a general anaesthetic and cardiopulmonary bypass. The options were put to our patient who consented to the 
procedure.

We took care to reduce fetal irradiation by limiting the exposure time and protecting the fetus with lead shields. The maximum fetal dose was estimated to be less than $0.7 \mathrm{mSv}$ during the angioplasty and less than $0.55 \mathrm{mSv}$ at the time of the initial cardiac catheterisation. This is well within the limits of acceptable risk to the fetus. The current consensus of expert opinion in the United States suggests that there is no reason for concern if the dose to the embryo is $<10 \mathrm{mSv}$ at any stage of the pregnancy. With exposure of less than $10 \mathrm{mSv}$, current evidence suggests that the risk of adverse effects (teratogenesis, carcinogenesis, and prenatal death) is small compared with the spontaneous incidence (4-6\%) of congenital abnormalities in liveborn infants with no history of irradiation. If the dose to the embryo was in the $10-100 \mathrm{mSv}$ range, radiation risk alone is not grounds for recommending therapeutic abortion. Only if the dose to the embryo was $>100 \mathrm{mSv}$ should therapeutic abortion be recommended on grounds of $x$ ray exposure alone. ${ }^{20}$ For comparison, the gonadal dose received with a routine abdominal radiograph or an intravenous urogram is $1 \mathrm{mSv}$ and $4 \mathrm{mSv}$ respectively.

There have been no studies to determine the optimal drug regimen after percutaneous transluminal coronary angioplasty nor is it known how long such a regimen is required. We routinely give heparin for 18-24 hours after angioplasty, particularly in patients with unstable angina, and maintain the patient on vasodilator (nitrates, calcium antagonists, or both) and antiplatelet drugs. $\beta$ Blockers are usually avoided. Administration of heparin during and after the angioplasty was considered to be relatively safe because heparin does not cross the placenta. ${ }^{21}$ Warfarin and other coumarin derivatives are teratogenic and should be avoided in the first trimester of pregnancy ${ }^{21-23}$, and if they are used later they should be stopped before labour to prevent excessive blood loss. Studies of an association between the ingestion of aspirin and the development of fetal malformations are inconclusive ${ }^{24-26}$; any possible association probably requires the ingestion of large doses of aspirin, as does the promotion of premature vasoconstriction of the ductus arteriosus, which can lead to increased fetal pulmonary artery pressure and, during long term treatment, disturbance in pulmonary vascular development. In our case we prescribed a low dose of aspirin; however, even this can cause minor haemorrhagic consequences in newborn infants. ${ }^{25}$ Dipyridamole, however, is not known to be associated with fetal malformations. ${ }^{27}$

Our patient had a limited myocardial infarct in mid-pregnancy. She survived cardiac arrest associated with ventricular fibrillation, which fortun- ately occurred when she was already on a coronary care unit. Subsequent unstable symptoms were found to be associated with a tight stenosis of the left anterior descending coronary artery that was particularly suited to treatment by percutaneous transluminal coronary angioplasty. Exposure of the fetus to $x$ rays was kept to a minimum. The application of this technique allowed the mother to have an angina free pregnancy, delivery, and puerperium, and she was delivered of a normal male infant. No decision has been reached regarding further pregnancies.

\section{References}

1 Ginz B. Myocardial infarction in pregnancy. J Obstet Gynecol 1970;77:610-5.

2 Hankins GDV, Wendel GD, Leveno KJ, Stoneham J. Myocardial infarction during pregnancy. Obstet Gynecol 1985;65:139-46.

3 Stokes IM, Evans J, Stone M. Myocardial infarction and cardiac arrest in the second trimester followed by assisted vaginal delivery under epidural analgesia at 38 weeks gestation. Case report. Br J Obstet Gynaecol 1984;91:197-8.

4 Shapiro S, Slone D, Rosenberg L, et al. Oral contraceptive use in relation to myocardial infarction. Lancet 1979;i:743-6.

5 Slone D, Kaufman DW, Shapiro S, et al. Risk of myocardial infarction in relation to current and discontinued oral contraceptive use. $N$ Engl J Med 1981;305:420-4.

6 Rosenberg L, Miller DR, Kaufman DW, et al. Myocardial infarction in woman under fifty years of age. $J A M A$ 1983;250:2801-6.

7 Lees KR, Rubin PC. Prescribing in pregnancy. Treatment of cardiovascular diseases. $\mathrm{Br}$ Med $J$ 1987; 294:358-60.

8 Pruyn SC, Phelan JP, Buchanan GC. Long-term propanolol therapy in pregnancy: maternal and fetal outcome. Am J Obstet Gynecol 1979;135:485-9.

9 Habib A, McArthy JS. Effects on the neonate of propanolol administered during pregnancy. $J$ Pediatr 1977;91:808-11.

10 Lieberman BA, Stirrat GM, Dohen SL, Beard RW, Pinker GD, Belsey E. The possible adverse effect of propranolol on the fetus in pregnancy complicated by severe hypertension. $\mathrm{Br} J$ Obstet Gynaecol 1978;85:678-83.

11 de Swiet $\mathrm{M}$. Antihypertensive drugs in pregnancy. $\mathrm{Br}$ Med J 1985;291:365-6.

12 Reynolds B, Butters L, Evans J, Adams T, Rubin PC. First year of life after the use of atenolol in pregnancy associated with hypertension. Arch Dis Child 1984;59:161-3.

13 Briggs GG, Freeman RK, Yaffe SJ. Drugs in pregnancy and lactation. Baltimore: Williams and Wilkins, 1986:311-2.

14 Lewis P. Clinical pharmacology in obstetrics. Bristol: Wright PSG, 1983:205-6.

15 Briggs GG, Freeman RK, Yaffe SJ. Drugs in pregnancy and lactation. Baltimore: Williams and Wilkins, 
1986:309-10 and 468-9.

16 British National Formulary 1986:No 12:18.

17 Sasse L, Wagner R, Murray FE. Transmural myocardial infarction during pregnancy. Am J Cardiol 1975;35:448-52.

18 Bernal JM, Miralles PJ. Cardiac surgery with cardiopulmonary bypass during pregnancy. Obstet Gynecol Surv 1986;41:1-6.

19 Majdan JF, Walinsky P, Cowchock S, Wapner RJ, Plazk $\mathrm{L}$. Coronary artery bypass surgery during pregnancy. Am J Cardiol 1983;52:1145-6.

20 Gibbs J. The risks of exposure in utero to X-radiation. In: Grainger RG, Allison DJ, eds. Diagnostic radiology. Edinburgh: Churchill Livingstone, 1986:1590.

21 Hall JG, Pauli RM, Wilson KA. Maternal and fetal sequelae of anticoagulation during pregnancy. Am J Med 1980;68:122-40.
22 Bloomfield DK. Fetal deaths and malformations associated with the use of coumarin derivatives in pregnancy. Am J Obstet Gynecol 1970;107:883-8.

23 Iturbe-Alessio I, del Carmen Fonseca M, Mutchinik O, Angel Santos M, Zajarias A, Salazar E. Risks of anticoagulant therapy in pregnant women with artificial heart valves. $N$ Engl J Med 1986;315:1390-3.

24 Howden $C W$. Prescribing in pregnancy. Treatment of common minor ailments. $\mathrm{Br} \mathrm{Med} J$ 1986;293: 1549-50.

25 Briggs GG, Freeman RK, Yaffe SJ. Drugs in pregnancy and lactation. Baltimore: Williams and Wilkins, 1986:26-9.

26 Lewis P. Clinical pharmacology in obstetrics. Bristol: Wright PSG, 1983:49-52.

27 Briggs GG, Freeman RK, Yaffe SJ. Drugs in pregnancy and lactation. Baltimore: Williams and Wilkins, 1986:149-50. 